\title{
Gesundheitskompetenz - wie viel darf's denn sein?
}

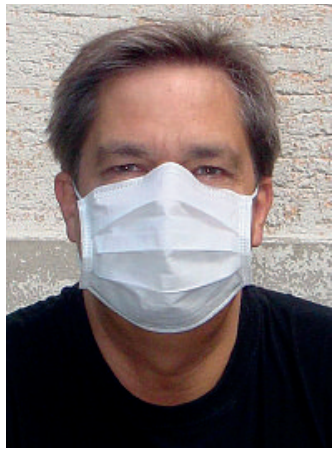

Eberhard Wolff
Die Präventivmedizin, vor allem die intervenierende, ist ein dynamisches Fach. Sie ist ständig auf der Suche nach verfeinerten Konzepten, wie man auf das Gesundheitsverhalten der Bevölkerung einwirken könne. Früher nannte man dies auch «Hygienische Volksbelehrung», und deren erhobener Zeigefinger war unübersehbar. In der verschärften Form arbeitete diese Gesundheitsaufklärung mit Angst, Abschreckung, negativen Emotionen. Der Lausanner Arzt Tissot etwa malte schon vor über 200 Jahren die Folgen des wahrhaft bedrohlichen Übels der «Selbstbefleckung» (sprich: Onanie) in Form von eindrucksvollen Schauergeschichten aus - mit zweifelhafter Wirkung. Einen Nachhall solch schwarzer Gesundheitspädagogik finden wir auch heute bisweilen noch auf Schweizer Plakatwänden.

Auch sanftere Methoden wie die nüchterne Gesundheitsinformation stehen unter dem Generalverdacht mangelhafter Effizienz. Die Präventivmedizin sinnt aber ständig auf Abhilfe. So erkannte sie, dass das erwünschte Gesundheitsverhalten in den Lebensstil der Angesprochenen passen muss. Auf Plakaten wurde den Drogen ein «uncooles» Image angehängt, auf dass Kiffen nicht mehr in den «Lifestyle» der Jugend passe.

Das neue Zauberwort heisst seit einigen Jahren «Health Literacy». Das meint den Kampf gegen den gesundheitlichen Analphabetismus, hierzulande mit «Gesundheitskompetenz» übersetzt. In schulischen Lehrplänen hat das Lernziel «Kompetenzerwerb» schon länger das Ziel des reinen Auswendiglernens abgelöst.

Unter dem trendigen Titel «Gesundheitskompetenz» stand denn auch die diesjährige Swiss Public Health Conference (www.public-health.ch). Ist das Konzept nun aber eine wichtige Innovation oder alter Wein in neuen Schläuchen, wie der Berner Präventivmediziner Thomas Abel auf der Tagung fragte?

Eine dreistufige Antwort gab ein Kenner der Materie, Don Nutbeam aus Southampton. In einem einfachen Sinn von «Functional Literacy» bedeute Gesundheitskompetenz lediglich die Fähigkeit, sich das notwendige Wissen über die Ursachen und Zusammenhänge von Krankheit und Gesundheit und die Wege dorthin anzueignen. So wichtig diese Erkenntnis ist, etwa für die Migrantenmedizin, so wenig neu ist sie.

Die höhere Kompetenzstufe der «Interactive Literacy» besteht nach Nutbeam in der Fähigkeit, die Bedeutung von Gesundheitsinformationen zu verstehen, sie einschätzen zu können und aktiv am Gesundheitssystem teilzuhaben. Ansätze hierzu gibt es reichlich und vielfältig. Ib Johansen aus Odense etwa berichtete auf dem Kongress von einem dänischen Projekt, in dem Patienten Einblick in ihr elektronisches Patien- tenjournal haben und selber online daran mitschreiben, etwa ihre Blutzuckerwerte eintragen. Patienten werden zu Partnern im therapeutischen Prozess. Der sich entwickelnde Gesundheitsmarkt wird die immer besser informierten Patienten ohnehin zunehmend als Kunden ansehen müssen, so Karin Frick vom Zukunfts-Think-Tank Gottlieb-Duttweiler-Institut.

Die am weitesten gehende Form von Gesundheitskompetenz besteht für Nutbeam in der «Critical Literacy». Auf dieser Stufe können Patienten sich eine Meinung bilden und den Nutzwert des Angebots für sich selbst einschätzen. Sie haben eine aktivere Rolle und eine grössere Kontrolle über die eigene Gesundheit.

Was aber, wenn die selbstverantwortlichen Patienten zu anderen Schlüssen kommen als die Therapeuten oder wohlmeinende Gesundheitsförderer? Was, wenn ein Migrant eben doch auf traditionelle Heilverfahren aus der Heimat schwört, die hierzulande weniger goutiert werden - es ist eben nicht alles so en vogue wie die TCM? Wird die Patientenautonomie noch als eigene Form von Kompetenz respektiert, wenn sie eigensinnig ist? Für Ilona Kickbusch, eine der Expertinnen des Modells, liegt darin ein «Dilemma», wie sie auf der Tagung bekräftigte.

Man kann ein Dilemma aber auch als Chance verstehen und sich die Patienten konsequent als Partner im Gesundheitswesen denken. Denn ohne eine grundsätzlich akzeptierte Möglichkeit zum Widerspruch läuft ein Konzept wie das der «Gesundheitskompetenz» Gefahr, letzten Endes doch nur eine Art paternalistischer Gesundheitserziehung zu sein, eine Art Fernsteuerung in den Köpfen willfähriger Patienten, mögen die Inhalte auch noch so gut gemeint sein.

Immerhin: Der Kongress genehmigte sich selbst ein Stück der subversiven «Critical Health Literacy», indem sich die Public-Health-Gemeinschaft den Event trotz Schweinegrippenwarnungen mit 450 potentiellen Infektionsherden leistete. Es sollen sogar Küsse getauscht worden sein! Den Zürcher Lehrern und Schulkindern in der Nachbarschaft war so viel Eigenverantwortung zur gleichen Zeit nicht gestattet. Sie mussten auf klassenübergreifende Aktivitäten verzichten. Schüler und Lehrer durften sich in den ersten Schuljahrswochen auch nicht mehr die Hand geben. Tissot hätte seine wahre Freude daran gehabt.

Zurück zur Eingangsfrage: Das Modell der Gesundheitskompetenz ist dann innovativ, wenn es Patientenkompetenz wirklich ernst nimmt. Und es ist eine Chance der intervenierenden Präventivmedizin, das alte Image der Gesundheitsbelehrung mit erhobenem Zeigefinger nach langer Zeit gänzlich abzustreifen.

Eberhard Wolff* 